\title{
Conservación en el Parque Nacional Arrecifes de Xcalak
}

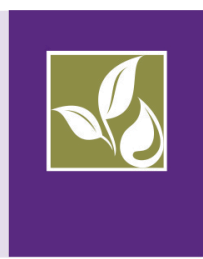

Recibido: 19/02/2016 · Aceptado: 07/04/2016

\author{
Leonardo Noriel López Jiménez* \\ Parque Nacional Arrecifes de Xcalak \\ Comisión Nacional de Áreas Naturales Protegidas
}

\section{Resumen}

Este trabajo abarca el proceso de creación del Parque Nacional Arrecifes de Xcalak como área natural protegida desde la iniciativa promovida por la comunidad de Xcalak de conservar los recursos naturales hasta la promulgación del decreto oficial en el año 2000. De igual forma se incluye la riqueza natural de los ecosistemas terrestres y marinos, y los servicios ambientales asociados a ellos que benefician a la sociedad, además de presentar las acciones de conservación que realizan los guardaparques para asegurar la prevalencia de estos recursos. Estas acciones se realizan mediante diferentes líneas de trabajo contenidas en el programa de manejo con el fin de proteger los recursos naturales para que la comunidad se beneficie a largo plazo y se fomente el desarrollo sustentable. Al final se mencionan los retos y oportunidades a los que esta área natural protegida se enfrenta para conseguir un manejo integral de los recursos de Xcalak.

Palabras clave: Manejo, sustentable, Conanp, arrecife, pesca.

*Correo electrónico: leonardonoriel@hotmail.com 


\title{
Conservation in the Arrecifes National Park of Xcalak
}

\author{
Leonardo Noriel López Jiménez* \\ Parque Nacional Arrecifes de Xcalak, \\ Comisión Nacional de Áreas Naturales Protegidas
}

\begin{abstract}
This paper covers the creation process of the Xcalak National Park as a protected natural area beginning with the Xcalak community initiative to conserve natural resources until its official decree in 2000 . The natural wealth of the land and marine ecosystems are included as are the environmental services and social benefits presenting the conservation actions performed by park rangers to ensure the prevalence of these natural resources. These actions are carried out through different lines of work contained in the management program to protect these natural resources to benefit the community on a longterm basis and encourage sustainable development. To conclude, the challenges and opportunities that are faced by this protected natural area to achieve an integral management of Xcalak resources are mentioned.
\end{abstract}

Key words: Management, sustainable, Conanp, reef, fishing.

*E-mail: leonardonoriel@hotmail.com 


\section{Introducción}

La Comisión Nacional de Áreas Naturales Protegidas (Conanp) es la institución encargada de conservar el patrimonio natural de México estableciendo áreas naturales protegidas (ANP), fomentando en las comunidades asentadas en su entorno una cultura de conservación y un desarrollo sustentable. El Parque Nacional Arrecifes de Xcalak (pNax) es una de las 177 Anp administradas por la Conanp, y se localiza en la parte sur de Quintana Roo, México, en el municipio de Othón P. Blanco. El PNAX y su zona de influencia cuentan con diversos ecosistemas terrestres y marinos en buen estado de conservación que son susceptibles de ser aprovechados de manera sustentable para el beneficio a largo plazo de las comunidades de la zona (Conanp, 2004). Esta Anp cubre una superficie total de 17949 ha; 13935 corresponden a ecosistemas marinos y 4543 a ecosistemas terrestres (Conanp, 2004). Actualmente las ANP son un instrumento importante en la política am-biental en México y el PNAx, junto con su programa de manejo, podrá garantizar la compatibilidad entre el aprovechamiento de los recursos naturales y su conservación. A continuación se presenta el proceso de creación de esta ANP, la importancia ambiental y económica de sus ecosistemas, y las acciones de conservación puestas en práctica para proteger estos recursos naturales de acuerdo con ciertas líneas de trabajo, así como los retos y oportunidades que enfrenta para generar un desarrollo sustentable.

\section{Inicio y creación}

La creación de esta ANP surgió como una inquietud de la comunidad de Xcalak para proteger y conservar los recursos naturales marinos y costeros, debido a que 1) los recursos pesqueros estaban en decline y 2) el gobierno estatal planteó que la zona sería destinada para el proyecto turístico Costa Maya (López-Santos, McCann, Molina-Islas y Rubinoff, 1997; McCann y Rubinoff, 1997; Bezaury-Creel et al., 1998; Robadue y Rubinoff, 2003; Daltabuit-Godás, Cisneros-Reyes y Valenzuela-Valdivieso, 2007); actualmente este proyecto continúa. En 1995 esta inquietud se consignó en dos cartas en las que se manifestaba el interés por un 
manejo sustentable de los recursos naturales, de parte de la Sociedad Cooperativa de Producción Pesquera "Andrés Quintana Roo"; una carta se entregó al Gobierno de Quintana Roo: ahí se solicitaba apoyo para conservar los recursos naturales y desarrollar actividades turísticas que fueran compatibles con la conservación; en ese mismo año, a través de la Delegación de Xcalak, se envió la segunda carta, dirigida a la Secretaría de Medio Ambiente, Recursos Naturales y Pesca, ahora Secretaría de Medio Ambiente y Recursos Naturales (Semarnat), que hacía énfasis en el interés de establecer un área natural protegida (López-Santos et al., 1997; McCann y Rubinoff, 1997).

Entre 1995 y 1997 la comunidad de Xcalak, con el apoyo de Amigos de Sian Ka'an y el Centro de Recursos Costeros de la Universidad de Rhode Island, realizó el diagnóstico ambiental y determinó los sitios de interés biológico, social y turístico del lugar (López-Santos et al., 1997; McCann y Rubinoff, 1997). Para coordinar todas estas actividades e impulsar el desarrollo de la propia comunidad, en 1996 se formó el Comité Comunitario para la Protección y el Manejo de los Recursos Costeros de Xcalak, integrado por el presidente de la Sociedad Cooperativa de Pesca, el sector turístico y el delegado de la comunidad (López-Santos et al., 1997; Conanp, 2004). A partir de ello, el comité comunitario organizó reuniones con el gobierno estatal y federal, así como con los sectores interesados, para diseñar y proponer acciones de manejo para el área protegida. Finalmente, el 5 de junio del 2000 se dio a conocer el aviso del decreto de la creación de esta nueva AnP y el 27 de noviembre de ese mismo año fue publicado en el Diario Oficial de la Federación el decreto de creación del Parque Nacional Arrecifes de Xcalak. La iniciativa de Xcalak es un ejemplo de los esfuerzos comunitarios que trabajan hacia el manejo de los recursos costeros en una forma integrada (McCann y Rubinoff, 1997).

\section{Riqueza natural y servicios ambientales}

La Península de Xcalak comprende algunas de las áreas costeras con menor desarrollo en México a pesar de que tiene hábitats como los manglares, grandes lagunas, sabanas, dunas costeras y playas íntimamente interconectados 
(Bezaury-Creel et al., 1998; Rosado et al., 1998) (figura 1). Esta pequeña área contiene un amplia variedad de especies y es extremadamente sensible a los cambios de uso de suelo (Merediz-Alonso y MacKinnon, 1997; Rosado et al., 1998). Los ambientes terrestres y marinos del pNAX resguardan ecosistemas y especies biológicamente importantes, son altamente productivos y las actividades socioeconómicas en Xcalak dependen de ellos. Al lograr un manejo adecuado, la comunidad se beneficiará de ellos a largo plazo.

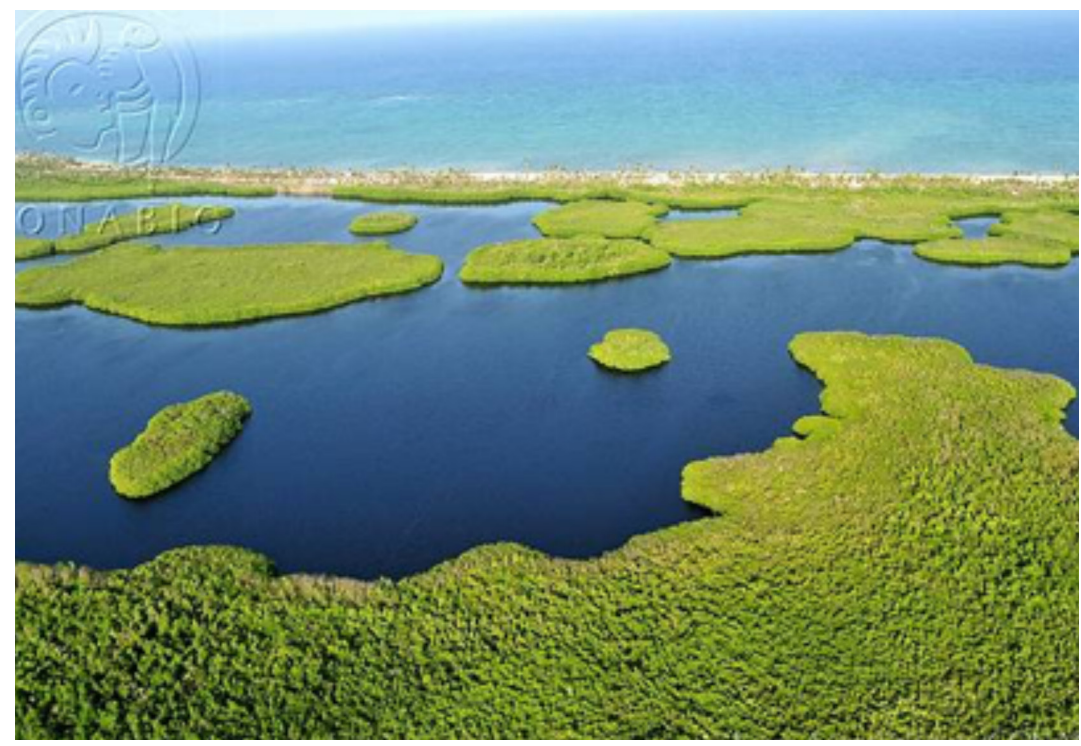

Fuente: Comisión Nacional para el Conocimiento y Uso de la Biodiversidad.

FiguRA 1. Ambientes costeros en Xcalak, al sur de Quintana Roo

Dentro del polígono terrestre del PNAX se encuentra un complejo lagunar integrado por las lagunas Huach, Tanquilá, Santa Rosa y Santa Julia, conectadas por canales y zonas de "bajos”; es esta la única zona en la costa sur de Quintana Roo que tiene comunicación superficial permanente con el mar a través del río Huach (López-Santos et al., 1997; Avilés-Torres, 2002). Estos complejos lagunares son sitios importantes para aves migratorias, y en ellos habitan las cuatro especies de mangle 
que predominan en México, cuya especie más abundante es el mangle rojo (Rhizophora mangle; Olivera-Gómez, 1996). Los manglares se presentan en asociaciones sobre la línea del litoral (Cabrera-Cano, 1997); su presencia impide el oleaje intenso y sirven de lugar para el refugio y crecimiento de juveniles de diversos peces e invertebrados marinos. En los ambientes terrestres existen dos tipos de selvas que dependen del grado de inundación que presenten durante la época de lluvias: selva baja caducifolia y selva baja inundable (Cabrera-Cano, 1997; Rosado et al., 1998). En estas selvas y lagunas es posible que habiten 155 aves, 27 anfibios y reptiles, y 31 mamíferos (Conanp, 2004), muy seguramente el jaguar (Panthera onca; Chávez y Zarza, 2009) y el tapir (Tapirus bairdii; Carrillo, Weissenberger y Reyna-Hurtado, 2015).

El sistema arrecifal del litoral de Xcalak forma parte del Sistema Arrecifal Mesoamericano (SAM) y se encuentra cercano a la costa en algunos sitios y hasta $1000 \mathrm{~m}$ mar adentro en otros, formando una laguna somera bien desarrollada de no más de cuatro metros de profundidad (García-Beltrán, 1997; Rosado et al, 1998; Hernández-Arana, 2010). La laguna arrecifal está constituida principalmente por fondos arenosos cubiertos por praderas de dos especies de pasto marino, el pasto manatí (Syringodium filiforme) y el pasto tortuga (Thalassia testudinum), y tiene parches coralinos conformados por Montastrea annularis principalmente (Olivera-Gómez, 1996; García-Beltrán, 1997; Kramer y Richards, 2002; Jordán-Dahlgren y Rodríguez-Martínez, 2003; Hernández-Arana, 2010). La vegetación sumergida en la laguna arrecifal está en buen estado de conservación y estas praderas se extienden hasta el arrecife (CalvaBenítez y Torres-Alvarado, 2011).

Los arrecifes de coral son los depósitos de biodiversidad más importantes en el ambiente marino (Calderón-Aguilera y Reyes-Bonilla, 2005), y los del PNAX albergan una gran diversidad de especies (figura 2). En este ecosistema habita la langosta del Caribe (Panulirus argus), de importancia comercial (Sosa-Cordero, 2011), el caracol rosado (Strombus gigas; Pool-Pool et al., 2011), en veda por cinco años (2012-2017), 98 especies de peces arrecifales, 79 especies de macroalgas bentónicas, 28 de corales blandos (Conanp, 2004), y al menos 43 especies de corales escleractinios o corales duros, como el lechuga (Agaricia agaricites), cerebro (Montastrea annularis, 


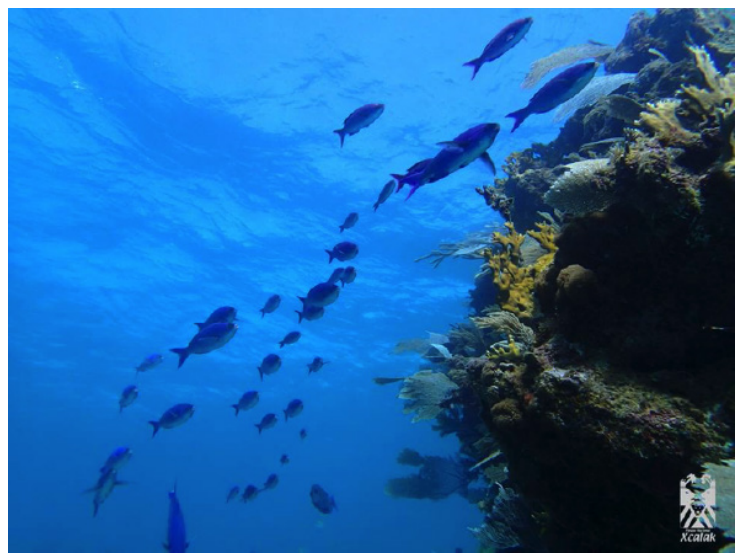

Figura 2. Arrecifes de coral en la zona núcleo en el norte del Parque Nacional Arrecifes de Xcalak

Fuente: Parque Nacional Arrecifes de Xcalak.

Diploria spp.), estrella (Siderastrea spp.), dedos (Porites spp.), cuerno de ciervo (Acropora cervicornis) y cuerno de alce (A. palmata; Conanp, 2004; Ruiz-Zárate y Arias-González, 2004); estos dos últimos corales son considerados los principales constructores del arrecife (Lighty, Macintyre y Stuckenrath, 1982). Además de ello, el pNAx resguarda el segundo parche de coral cuerno de alce más grande de Quintana Roo (García-Salgado, NavaMartínez y Samos-Falcón, 2013) (figura 3).

FIGURA 3. En la imagen se aprecia al coral cuerno de alce en el PNAx, una de las especies ecológicamente importantes por participar en la formación inicial de los arrecifes de coral

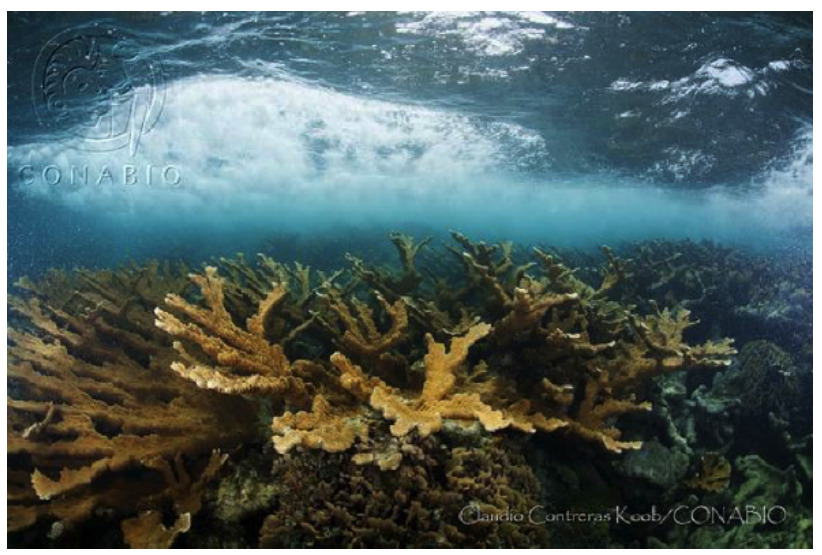

Fuente: Comisión Nacional para el Conocimiento y Uso de la Biodiversidad. 
Las agregaciones reproductivas de peces arrecifales ocurren en momentos y lugares específicos (Domeier y Colin, 1997). A lo largo de la costa de Quintana Roo se han localizado sitios de agrupaciones reproductivas de mero (Ephinephelus striatus) y en el PNAx se encuentran cinco de ellos (Sosa-Cordero, Medina-Quej, Herrera y Aguilar-Dávila, 2002); el de Punta Gavilán es el único verificado científicamente y se encuentra en buen estado de conservación (Medina-Quej et al., 2004); es conocido por los pescadores desde hace casi veinte años (Bolio-Moguel, 2007). Frente a la comunidad de Xcalak se localiza una formación coralina atípica para los arrecifes de Quintana Roo, localmente conocida como "La Poza” (López-Santos et al., 1997). Esta formación es un canal de arena alargado con una profundidad de hasta $30 \mathrm{~m}$ y un ancho de 400 m (Hernández-Arana, 2010) y es el principal sitio atractivo para el buceo por su belleza escénica, pues puede observarse a un gran número de sábalos (Megalops atlanticus), el pez emblemático de la comunidad (figura 4).

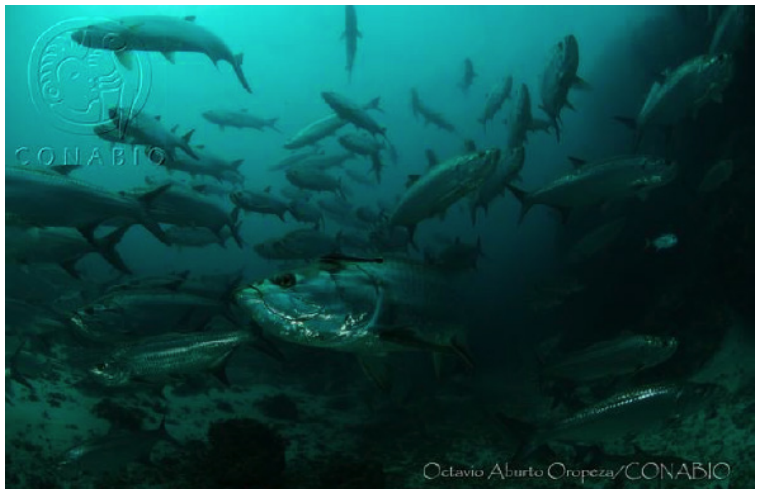

Fuente: Comisión Nacional para el Conocimiento y Uso de la Biodiversidad.
Figura 4. El sábalo es la especie emblemática de la comunidad de Xcalak; habita en La Poza en el Parque Nacional Arrecifes de Xcalak

La importancia de los ecosistemas arrecifales radica en sus funciones ecológicas, económicas y culturales (Crosby, Brighouse y Pichon, 2002). Los arrecifes de coral están reconocidos por su enorme diversidad biológica, porque contribuyen a la protección costera y porque son sitios de importancia económica, ya que ofrecen recursos pesqueros y atractivos turísticos (Calderón-Aguilera y Reyes-Bonilla, 2005). La pesca 
comercial y el turismo de bajo impacto son las principales actividades económicas de la población en Xcalak (Daltabuit-Godás, Vázquez, Cisneros y Ruiz, 2006; Thomassiny-Acosta, 2010). Los arrecifes del PNAX permiten actividades turísticas como buceo libre, buceo autónomo, recorridos en embarcaciones motorizadas y no motorizadas y pesca deportiva, y benefician a las pesquerías porque sirven de protección a los peces juveniles y proveen especies de uso comercial, como la langosta, el mero y sus especies asociadas (Medina-Quej et al., 2006). La pesca está organizada a través de la sociedad cooperativa y el turismo es una actividad que aún no despunta, aunque en el futuro pudiera darse un desarrollo por el corredor turístico Costa Maya (Daltabuit-Godás et al., 2007); estas dos actividades económicas dependen directamente de la presencia del arrecife (Thomassiny-Acosta, 2010).

El PNAX representa una oportunidad para generar programas y proyectos educativos ambientales sobre la importancia biológica y económica de los recursos naturales, dirigidos principalmente a la población de Xcalak. Además, su riqueza biológica marina y terrestre ha llamado la atención a nivel internacional, ya que el área está incluida como sitio prioritario para el SAM y sus humedales están reconocidos a nivel internacional desde 2003 por la Convención Ramsar. La dirección del PNAX tiene la misión de proteger la diversidad biológica de esta zona y fomentar el desarrollo sustentable mediante diversas acciones de conservación, para permitir el disfrute de los servicios ambientales que brindan estos ecosistemas.

\section{Acciones de conservación}

La Conanp tiene presencia permanente en el PNAX gracias a la adquisición de equipamiento necesario, a la contratación de personal y a la construcción de instalaciones adecuadas para realizar y dar seguimiento a los proyectos de conservación y manejo dentro del ANP a partir del 2004 (Hadad-López, 2010). El Programa de Manejo es el instrumento principal de regulación y de planeación; en él se establecen las actividades, acciones y lineamientos básicos para el manejo y la administración del ANP, de acuerdo con el Reglamento de la Ley General 
de Equilibrio Ecológico y Protección al Ambiente en materia de áreas naturales protegidas. El PNAX tiene su programa de manejo desde 2004, el cual contiene las estrategias y acciones para garantizar el manejo, el uso sustentable, la conservación, la protección y la restauración de los recursos costeros y marinos (Conanp, 2004); lo anterior ocurre mediante las siguientes líneas de trabajo como estrategias de conservación:

- Inspección y vigilancia. Las reglas administrativas establecidas en el programa de manejo son obligatorias para todas las personas que realicen cualquier tipo de actividad dentro del PNAX. Por ello la dirección se coordina estrechamente con la Secretaría de Marina, la Procuraduría Federal de Protección al Ambiente, la Procuraduría General de la República y la Comisión Nacional de Pesca para vigilar el área a través de actividades preventivas y correctivas y evitar el deterioro ambiental ajustándose a la normatividad correspondiente. En este sentido, una parte importante de este trabajo es mantener informados a los usuarios para evitar alguna acción no permitida.

- Investigación. La investigación científica es indispensable para mejorar las estrategias de conservación y encontrar alternativas de uso sustentable. El PNAX es un laboratorio natural parcialmente conocido, e incluso desconocido en algunos aspectos. A pesar de ello, existen instituciones académicas y centros de investigación que desarrollan proyectos dentro del PNAX para aportar información que sirva en la toma de decisiones. A su vez se promueve y se dirige la investigación para que ayude a resolver la problemática en materia de pesquerías, ecología de arrecifes y especies invasoras principalmente. Debido a esto, se ha mantenido una relación constante con el Instituto Tecnológico de Chetumal (IтCH), El Colegio de la Frontera Sur (Ecosur) Unidad Chetumal y el Centro de Investigación y de Estudios Avanzados (Cinvestav) Unidad Mérida. Cabe mencionar que estas no son las únicas unidades académicas que realizan investigación en el ANP y que inclusive se pueden mantener y aumentar estas relaciones académicas e interactuar con otras entidades.

- Monitoreo. Como la pesca y el turismo son las principales actividades económicas en el PNAX (Thomassiny-Acosta, 2010), es necesario detectar 
su impacto en los ecosistemas mediante monitoreos sistemáticos. Dentro de los programas de monitoreo se incluye la evaluación de los principales recursos pesqueros, como la langosta del caribe, el caracol rosado (figura 5) y las agregaciones de mero, y los ecológicamente importantes, como la salud de los arrecifes, el pez león, las tortugas marinas y la fauna terrestre. Para llevar a cabo estas acciones, en años recientes se ha involucrado a jóvenes de la población para que participen en estas labores de conservación. El grupo se llama Jóvenes por Xcalak y está integrado por hombres y mujeres de entre 14 y 25 años de edad, los cuales han sido capacitados en técnicas de buceo, en el registro y la captura de datos biológicos enfocados en ambientes marinos y en la identificación taxonómica de especies marinas.

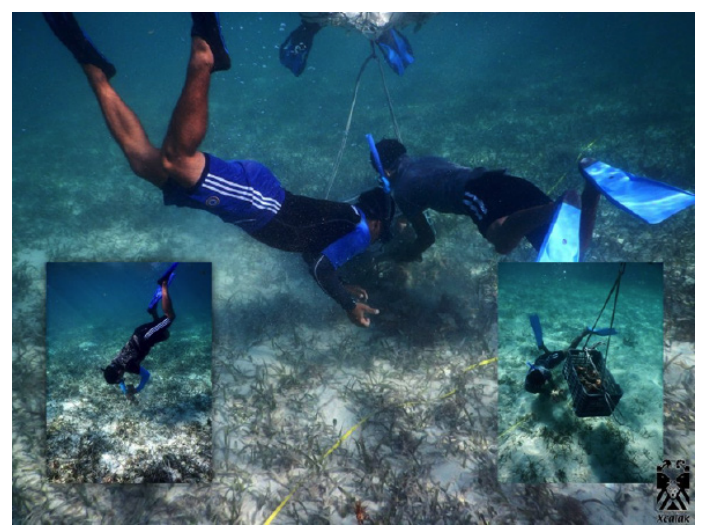

Fuente: Parque Nacional Arrecifes de Xcalak.
FigURA 5. El monitoreo biológico de caracol rosado en el PNAX consiste en el conteo y la medición de todos los individuos encontrados en cierta área. Esta acción la lleva a cabo el grupo Jóvenes por Xcalak (algunos de sus miembros se observan en la fotografía)

- Turismo. Las actividades turísticas requieren autorizaciones emitidas por la Conanp, por ser zonas exclusivas; se ha establecido el cobro de derechos por ingresar al ANP y existe señalización marina y terrestre que sitúa e informa a los turistas sobre el lugar en el que se encuentran, da información sobre las actividades permitidas y los sensibiliza sobre temas ambientales con el fin de prevenir el deterioro del entorno. De igual manera se capacita a los prestadores de servicios turísticos y a los guías locales en el conocimiento del área y las reglas administrativas para que 
sean ellos quienes repliquen esta información con las personas que visitan el PNAX con fines recreativos y turísticos (figura 6).

Figura 6. El curso de guías y capitanes se imparte a los prestadores de servicios turísticos del PNAX para reforzar el conocimiento sobre las ANP en México

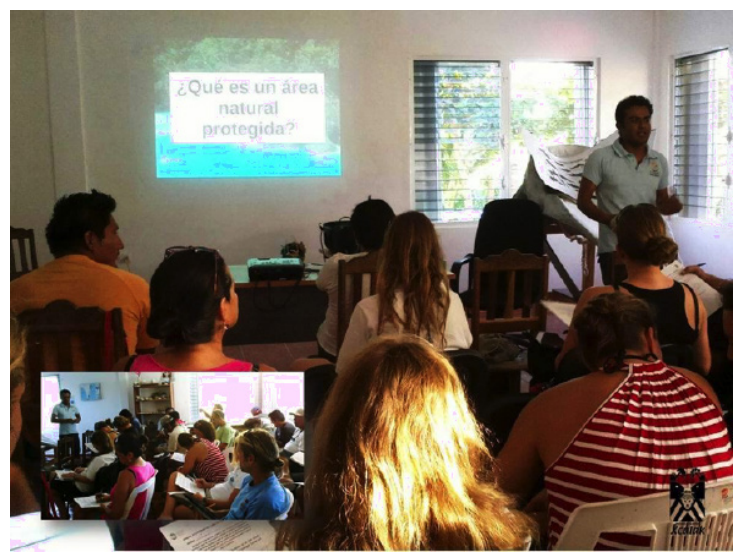

Fuente: Parque Nacional Arrecifes de Xcalak.

- Pesquerías. La pesca es la principal actividad económica en Xcalak (Thomassiny-Acosta, 2010) y está regulada por la Secretaría de Agricultura, Ganadería, Desarrollo Rural, Pesca y Alimentación, mediante su delegación estatal, y la Comisión Nacional de Pesca. La pesca de escama (pargo, huachinango, mojarra, liseta, mero) que realizan los pobladores de Xcalak es principalmente para autoconsumo, y el aprovechamiento de la langosta es de tipo comercial (Medina-Quej et al., 2006; Thomassiny-Acosta, 2010; Cal-Durán, 2014). Se fomenta que la pesca se apegue al marco legal y que sea sustentable, a través de la capacitación e implementación de prácticas pesqueras adecuadas en los ecosistemas arrecifales, y a su vez, se promueve la diversificación de actividades económicas.

- Programas de conservación. Con la implementación del Programa de Conservación para el Desarrollo Sostenible (Procodes), el Programa de Empleo Temporal (РЕT) y el Programa de Vigilancia Comunitaria (Provicom) se han generado oportunidades productivas y actividades complementarias para la población de Xcalak en favor de la conservación de los recursos naturales del PNAX (figura 7). Cada año se beneficia a más 
de cien personas entre pescadores, guías de turistas, artesanas, amas de casa y jóvenes, y al mismo tiempo, se disminuye la presión hacia los ecosistemas en la zona. Por su parte, el Programa de Conservación de Especies en Riesgo (Procer) coordina proyectos para recuperar las poblaciones de especies prioritarias de conservación.

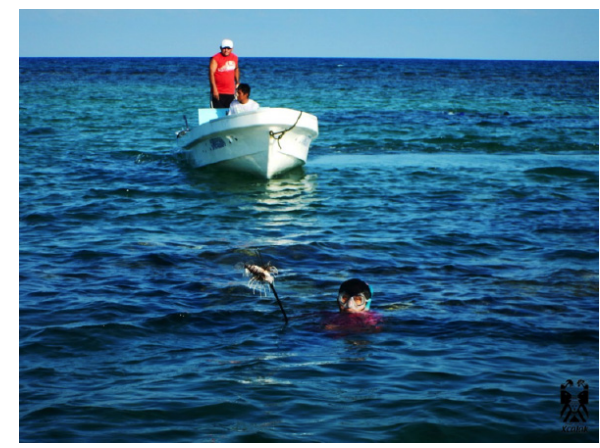

Fuente: Parque Nacional Arrecifes de Xcalak.

FiguRa 7. La implementación del Programa de Empleo Temporal para el control del pez león en el PNAX, con la participación de pescadores de Xcalak, es una acción que ayuda a mantener los ecosistemas sanos.

- Participación institucional y participación social. El manejo participativo se logra cuando los diferentes actores logran alianzas para compartir las responsabilidades y la toma de decisiones en el manejo de los recursos naturales (Borrini-Fereyabend, 1997). El consejo asesor es una herramienta que ayuda a la dirección del PNAX en la toma de decisiones en el manejo del área y actualmente incluye la representación de los tres órdenes de gobierno, de la comunidad, de las organizaciones de la sociedad civil, del grupo de investigadores, del sector pesquero y el turístico, y el grupo de jóvenes. Durante el año se realizan reuniones periódicas para tratar asuntos relevantes del área, lo que propicia una coordinación entre ellos y mantiene una comunicación oportuna y precisa. Es necesario remarcar que la conservación es inviable sin la participación de las comunidades humanas, ya que son parte importante de los ecosistemas (O’Neill, 2001), por ello este consejo asesor está establecido desde 2002 (López-Santos, 2003). 
- Educación y cultura ambiental. La educación ambiental es una herramienta ampliamente usada en el PNAX para apoyar a la conservación. Esta línea trabaja para lograr que la sociedad valore la biodiversidad, participe activamente en el manejo de los recursos naturales y se sensibilice en temas ambientales mediante pláticas, visitas guiadas, talleres educativos y de capacitación, intercambios de experiencias y otras actividades dirigidas a grupos de distintos niveles escolares y diferentes sectores sociales (figura 8). Se pretende que el PNAx logre influir en un modo positivo en el manejo de las principales problemáticas que impactan al área y que la población local se involucre y se vincule con el trabajo de conservación, razón por la cual muchos esfuerzos se dirigen a la gente en Xcalak, sin descuidar la realización de actividades en otros lugares y sectores. Así se podrá mantener y, eventualmente, incrementar su valor actual y potencial.

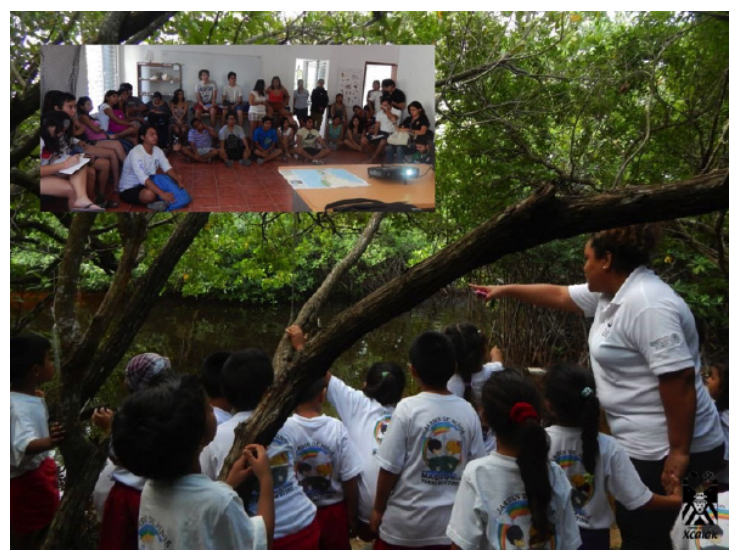

FIGURA 8. La educación ambiental es una de las herramientas fundamentales de la conservación. En la imagen se observa a un grupo de niños de preescolar conociendo los ecosistemas del PNAX y a un grupo de estudiantes de licenciatura conociendo las acciones de conservación.

Fuente: Parque Nacional Arrecifes de Xcalak.

- Difusión. El pnax da a conocer a los usuarios todas aquellas actividades de conservación que se lleven a cabo en el área protegida, así como los valores, cuidados y documentos que rigen su aprovechamiento, usando diferentes foros y medios. Actualmente el PNAX tiene presencia en dos redes sociales con el fin de cumplir con este objetivo: mediante Facebook 
se tiene una interacción directa y dinámica con los usuarios a través de las herramientas de esta red social (figura 9), y NaturaLista ofrece a los seguidores una galería de imágenes sobre su diversidad biológica. Así se pone de manifiesto la importancia de los ecosistemas del PNAX.

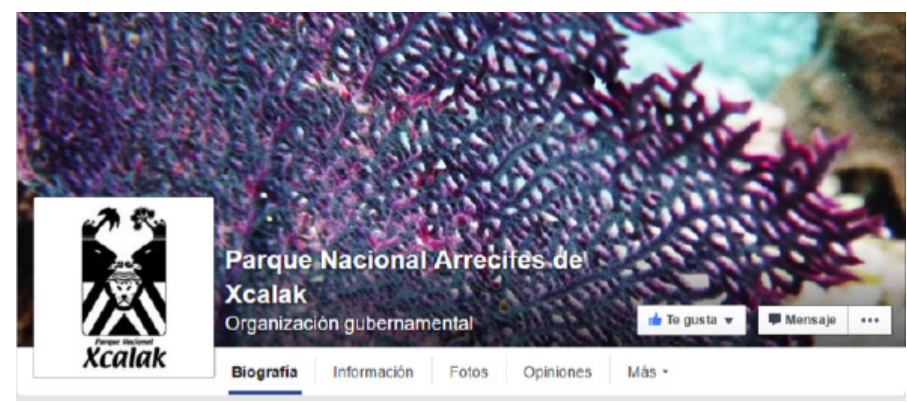

FiguRA 9. www.facebook.com/PNArrecifesdeXcalak

Las tareas de conservación en el PNAX son llevadas a cabo por un grupo interdisciplinario de especialistas y técnicos de campo dedicados a la gestión ambiental: los guardaparques. Ellos deben tener una visión amplia de la conservación que abarque aspectos socioculturales, económicos y ambientales de los recursos naturales, y son un nexo importante entre la comunidad de Xcalak y la conservación: garantizan que se resguarden los valores y elementos naturales, así como los valores culturales asociados a estos.

\section{Retos y oportunidades para un desarrollo sustentable}

Las actividades humanas ejercen una constante presión en los ambientes marinos y costeros dentro del polígono del PNAX y en su zona de influencia. Aunque las actividades acuáticas recreativas y el desarrollo turístico costero (cambio de uso de suelo y construcción de infraestructura) son unas de las principales amenazas para la conservación de los ecosistemas marinos en las áreas protegidas de México (Kramer y Richards, 2002) y en la costa de Quintana Roo (DaltabuitGodás et al., 2006), la actividad turística todavía no representa una amenaza para los ecosistemas en Xcalak. El desarrollo turístico en esta zona aún es 
incipiente (Daltabuit-Godás et al., 2006), aunque se prevé que el proyecto Costa Maya se extienda hasta Xcalak (González-Damián, 2011). Por ello, es necesario seguir regulando el turismo, ordenar las actividades de este tipo y crear un producto turístico alineado con la conservación; a su vez, tendrá que desarrollarse una oferta innovadora y de calidad de productos turísticos diversificados para reducir la presión en un atractivo natural específico. Esto representa una excelente oportunidad para el diseño y la implementación de estrategias que garanticen un desarrollo turístico sustentable.

A lo largo del tiempo y de manera tradicional, los pescadores han aprovechado los recursos pesqueros (Cal-Durán, 2014), y este parece ser el principal problema al que debe enfrentarse el PNAX. La pesca ilegal es un problema continuo cuya magnitud se desconoce, pero se sabe que ocurre durante todo el año. El uso de artes de pesca no sustentables y la extracción de recursos pesqueros en veda en zonas y temporadas no permitidas, son los principales problemas asociados con la pesca en el pNAX.

Tanto la pesca como el turismo son actividades económicas que representan una oportunidad de desarrollo sustentable que permita mantener las comunidades marinas y propicie el crecimiento económico y social de Xcalak. Por ello es necesario apoyar, fortalecer y consolidar estas actividades productivas dirigiéndolas a mercados justos que promuevan la conservación, implementar instrumentos económicos y financieros, y promover capacitaciones adecuadas en coordinación con otros actores. Además, se requiere brindar asistencia técnica dirigida a consolidar proyectos que incrementen el valor de los productos pesqueros y turísticos con innovación y tecnología. Asimismo es fundamental abordar y solucionar estas problemáticas de manera conjunta mediante una coordinación efectiva entre los usuarios, los tres órdenes de gobierno y las organizaciones de la sociedad civil.

El fortalecimiento de la participación social y el involucramiento de la comunidad de Xcalak en las actividades de conservación es importante para incrementar su papel en la gestión ambiental. Es necesario fomentar la sensibilización y aumentar la participación de la comunidad de Xcalak en la gestión de sus recursos ambientales, así como impulsar una cultura de la conservación de los recursos naturales para que los habitantes puedan reconocer su identidad, su cultura y sus intereses. La creación de un centro de interpretación ambiental 
servirá para promover la conservación de la vida silvestre de Xcalak utilizando herramientas y experiencias sensoriales para transmitir la información de manera que el visitante pueda sensibilizarse. Al mismo tiempo, podrán planificarse y controlarse las actividades de difusión, recreativas, turísticas y deportivas del PNAX. El desarrollo de este centro de interpretación debe considerar las necesidades de comunicación de los grupos de personas a los cuales estaría destinado.

La flora y la fauna de la zona han atraído a algunos estudiosos para realizar investigación; a pesar de ello, aún se está lejos de tener inventarios biológicos completos para los grupos taxonómicos del PNAX, así que se requiere fomentar proyectos que enriquezcan y actualicen los existentes. Hay una oportunidad para desarrollar investigación innovadora y de calidad, la información que se genere podrá ser usada en la toma de decisiones acerca del manejo de los recursos naturales y en la creación de proyectos y negocios productivos sustentables. Al mismo tiempo, será necesario crear convenios de colaboración entre universidad y centros de investigación regionales y nacionales para impulsar la investigación aplicada en conservación de la biodiversidad, y mejorar la transferencia y el intercambio de los resultados de estas investigaciones.

La acumulación de residuos sólidos traídos por las corrientes marinas a las costas del PNAX es un problema recurrente, sumado a la falta de tratamiento de aguas residuales y a la incorrecta disposición final de los residuos generados en la propia comunidad de Xcalak. Se requiere infraestructura para el tratamiento de estos residuos y de las aguas residuales, los cuales representan una fuente de contaminación para los manglares y el arrecife.

El manejo de las AnP requiere fondos económicos suficientes, oportunos y manejados de una forma eficiente; la falta de esta financiación y de recursos económicos es una gran amenaza para su conservación y protección (BezauryCreel y Gutiérrez-Carbonell, 2009). Para enfrentar estos retos y mantener las acciones de conservación, los esquemas de financiamiento del PNAX deben continuar promoviendo la coexistencia armónica de la pesca y el turismo (al ser las principales actividades económicas en Xcalak) y enfocarse a la sustentabilidad, así como asegurar la colaboración de los diferentes aliados locales, nacionales e internacionales.

Xcalak necesita un modelo de desarrollo sustentable integral, que incluya aspectos urbanos y de infraestructura adecuados al contexto natural y cultural, un uso racional de los recursos energéticos e hídricos, un adecuado manejo y 
disposición final de residuos y aguas residuales, así como generar un bienestar social y económico para la población. La base de todo esto es el patrimonio natural. La falta de ingresos económicos justos causa daños al ambiente, ya que impide ver la repercusión a largo plazo de la incorrecta explotación de los recursos naturales; a consecuencia de esto, hay más pobreza y más daños al ambiente. La conservación debe estar ligada con este modelo de desarrollo en Xcalak, lo que permitirá cambiar la situación actual. Al ser este un modelo integral, deberá contar con la participación de diferentes instituciones de gobierno que abarquen los aspectos mencionados, así como organizaciones de la sociedad locales que tengan incidencia en esos temas y, sobre todo, la participación activa de los usuarios y de la comunidad de Xcalak.

\section{Conclusiones}

La permanencia de la riqueza biológica del PNAX dependerá en buena medida de la implementación de correctas acciones de conservación y de la toma de decisiones que integren el conocimiento científico y el tradicional de la vida silvestre. Para lograr esto es necesario buscar mecanismos para promover que los pobladores de Xcalak se apropien de los recursos naturales y adopten alternativas de uso sustentable y, que al mismo tiempo, representen una opción real para su desarrollo. De igual forma es necesario compartir la responsabilidad de la conservación de la biodiversidad entre los actores gubernamentales, privados y de la sociedad civil para lograr una sinergia en el manejo integral de los recursos marinos y costeros de Xcalak. Es así como el PNAX sería considerado un ANP de excelencia.

\section{Fuentes consultadas}

Avilés-Torres, S. (2002). Influencia de la heterogeneidad espacial y la fluctuación climática en la comunidad de peces del sistema lagunar Río Huach, sur de Quintana Roo, México (Tesis de maestría). Centro de Investigación Científica y de Educación Superior de Ensenada, Ensenada.

Bezaury-Creel, J. y Gutiérrez-Carbonell, D. (2009). Áreas naturales protegidas y desarrollo social en México. En Conabio, Capital natural de México, vol. 
II: Estado de conservación y tendencias de cambio (pp. 385-431). México: Comisión Nacional para el Conocimiento y Uso de la Biodiversidad. Bezaury-Creel, J.E., López-Santos, C., McCann, J., Molina-Islas, C., Carranza, J., Rubinoff, P., ... Hale, L. (1998). Participatory coastal and marine management in Quintana Roo, Mexico. En International Tropical Marine Ecosystems Management Symposium. Simposio llevado a cabo en Townsville, Australia.

Bolio-Moguel, K. M. (2007). Caracterización y descripción pesquera del sitio de agrupación de peces "El blanquizal-Santa Julia", Quintana Roo, México: Invierno 2004-Primavera 2005 (Tesis de maestría). El Colegio de la Frontera Sur, Chetumal.

Borrini-Fereyabend, G. (1997). Manejo participativo de áreas protegidas: adaptando el método al contexto. Temas de política social. Quito: Unión Internacional para la Conservación de la Naturaleza-América del Sur.

Cabrera-Cano, E. F. (1997). La vegetación en la zona de Xcalak. Boletín Sian Ka'an, 17, 24-27.

Cal-Durán, C. M. (2014). Manejo tradicional de los ecosistemas costeros del sur de Quintana Roo (Tesis de licenciatura). Universidad de Quintana Roo, Chetumal.

Calderón-Aguilera, L. E. y Reyes-Bonilla, H. (2005). Arrecifes, una interacción de bella complejidad. Ciencia y Desarrollo, 30, 7-11.

Calva-Benítez, L. G. y Torres-Alvarado, R. (2011). Carbono orgánico y características texturales de sedimentos en áreas de pasto marino Thalassia testudinum en ecosistemas costeros del sureste del Golfo de México. Universidad y Ciencia, 27(2), 133-144.

Carrillo, N., Weissenberger, H. y Reyna-Hurtado, R. (2015). Distribución potencial del tapir centroamericano en la península de Yucatán. Therya, 6(3), 575-596.

Chávez, C. y Zarza, H. (2009). Distribución potencial del hábitat del jaguar y áreas de conflicto humano-jaguar en la Península de Yucatán. Revista Mexicana de Mastozoología, 13, 46-62.

Conanp (Comisión Nacional de Áreas Naturales Protegidas). (2004). Programa de Manejo del Parque Nacional Arrecifes de Xcalak. México: Autor.

Crosby, M. P., Brighouse, G. y Pichon, M. (2002). Priorities and strategies for 
addressing natural and anthropogenic threats to coral reefs in Pacific Island Nations. Ocean and Coastal Management, 45, 121-137.

Daltabuit-Godás, M., Cisneros-Reyes, H. y Valenzuela-Valdivieso, E. (2007). Globalización y sustentabilidad. El turismo en el sur de Quintana Roo. México: Centro Regional de Investigaciones Multidisciplinarias-Universidad Nacional Autónoma de México.

Daltabuit-Godás, M., Vázquez, L. M., Cisneros, H. y Ruiz, G. A. (2006). El turismo costero en la ecorregión del Sistema Arrecifal Mesoamericano. Cuernavaca: Centro Regional de Investigaciones MultidisciplinariasUniversidad Nacional Autónoma de México.

Domeier, M. L. y Colin, P. L. (1997). Tropical reef fish spawning aggregations: defined and reviewed. Bulletin of Marine Science, 60, 698-726.

García-Beltrán, G. (1997). Los arrecifes de Xcalak. Boletín Sian Ka’an, 17, 43-44. García-Salgado, M. A., Nava-Martínez, G. y Samos-Falcón, E. (2013). Identificación y caracterización de sitios de distribución de las poblaciones de Acropora palmata y Acropora cervicornis del Área Marina Prioritaria Xcalak-Mahahual (Reporte técnico final). México: Secretaría de Medio Ambiente y Recursos Naturales/Consejo Nacional de Ciencia y Tecnología/Oceanus.

González-Damián, A. (2011). Uso turístico de los recursos naturales. En C. Pozo, N. Armijo-Canto y S. Calmé (eds.), Riqueza biológica de Quintana Roo. Un análisis para su conservación, vol. 1 (pp. 197-201). México: El Colegio de la Frontera Sur/Comisión Nacional para el Conocimiento y Uso de la Biodiversidad/Gobierno del Estado de Quintana Roo/Programa de Pequeñas Donaciones.

Hadad-López, W. (2010). Análisis sistémico de la comunidad de Xcalak y el diseño y evolución de un área natural protegida: Parque Nacional Arrecifes de Xcalak (Tesis de maestría). Universidad Iberoamericana, Puebla.

Hernández-Arana, H. (2010). Caracterización del anp Parque Nacional Arrecifes de Xcalak y formulación de un programa de monitoreo (Informe técnico final sNib-Conabio proyecto No. DM015). México: El Colegio de la Frontera Sur.

Jordán-Dahlgren, E. y Rodríguez-Martínez, R. E. (2003). The atlantic coral reef ecosystem of México. En J. Cortés (ed.), Latin America coral reefs (pp. 131-158). Ámsterdam: Elsevier Science. 
Kramer, P. y Richards, P. (2002). Ecoregional conservation planning for the Mesoamerican Caribbean Reef. Washington, D. C: World Wildlife Fund.

Lighty, R. G., Macintyre, I. G. y Stuckenrath, R. (1982). Acropora palmata reef framework: a reliable indicator of sea level in the western Atlantic for the past 10,000 years. Coral Reef, 1, 125-130.

López-Santos, C. (2003). Análisis de la aplicación del manejo integral de recursos costeros. Boletín Sian Ka'an, 8-10.

López-Santos, C., McCann, J., Molina-Islas, C. y Rubinoff, P. (1997). Estrategia comunitaria para el manejo de la zona de Xcalak, Quintana Roo, México. Mérida: Comité Comunitario para la Protección y Manejo de los Recursos Costeros de Xcalak/Amigos de Sian Ka'an/Centro de Recursos Costeros de la Universidad de Rhode Island.

McCann, J. y Rubinoff, P. (1997). Una herramienta efectiva para promover el uso sustentable de los recursos costeros: el manejo costero integrado. Boletín Sian Ka'an, 17, 5-10.

Medina-Quej, A., Herrera-Pavón, R., Poot-López, G., Sosa-Cordero, E., BolioMoguel, K. M. y Hadad, W. (2004). Estudio preliminar de la agregación del mero Epinephelus striatus en "El Blanquizal" en la costa sur de Quintana Roo, México. Proceeding of the Gulf and Caribbean Fishering Institute, 55, 557-569.

Medina-Quej, A., Herrera-Pavón, R. L., Aguirre-García, B., Oliva-Rivera, J. J., Bolio-Moguel, K. M., Rodríguez-Canto, S. y Dzul-Panti, A. (2006). Diagnóstico de las pesquerías artesanales en el polígono del Parque Nacional Arrecifes de Xcalak (Informe técnico final). El Colegio de la Frontera Sur/Amigos de Sian Ka'an.

Merediz-Alonso, G. y MacKinnon, B. (1997). La fauna silvestre de Xcalak. Boletín Sian Ka'an, 17, 31-37.

O'Neill, R. V. (2001). Is it time to bury the ecosystem concept? (with full military honors, of curse!). Ecology, 82, 3275-3284.

Olivera-Gómez, A. Y. (1996). Modelo de ordenamiento ecológico de la zona sur de la península de Yucatán, Quintana Roo (Tesis de maestría). El Colegio de la Frontera Sur, Chetumal. 
Pool-Pool, F., Medina-Quej, A., Castro-Pérez, J. M., Gómez-Poot, J., HadadLópez, W. y de Jesús-Navarrete, A. (2011). Distribución y abundancia del Caracol rosado (Strombus gigas), en el Parque Nacional Arrecifes de Xcalak (pnax), Quintana Roo, México. Proceeding of the Gulf and Caribbean Fisheries Institute, 64, 391-395.

Robadue, D. D. y Rubinoff, P. (2003). Capacity building and strategic innovation: conserving critical coastal ecosystems in Mexico 1996-2003 (Coastal Management Report \#224). Narragansett: Coastal Reources CenterUniversity of Rhode Island.

Rosado, F. et al. (1998). Programa de Ordenamiento Ecológico Territorial de la Región Costa Maya. Chetumal: Universidad de Quintana Roo.

Ruiz-Zárate, M. A. y Arias-González, J. E. (2004). Spatial study of juvenile corals in the Northen region of the Mesoamerican Barrier System (MBRs). Coral Reef, 23, 584-594.

Sosa-Cordero, F. E. (2011). La langosta. Pesquería emblemática de Quintana Roo. En C. Pozo, N. Armijo-Canto y S. Calmé (eds.), Riqueza biológica de Quintana Roo. Un análisis para su conservación, vol. 1 (pp. 221227). México: El Colegio de la Frontera Sur/Comisión Nacional para el Conocimiento y Uso de la Biodiversidad/Gobierno del Estado de Quintana Roo/Programa de Pequeñas Donaciones.

Sosa-Cordero, E., Medina-Quej, A., Herrera, R. y Aguilar-Dávila, W. (2002). Agregaciones reproductivas de peces en el Sistema Arrecifal Mesoamericano: Consultoría Nacional-México (Informe preparado para el consultor internacional, Research Planning, y Proyecto sam-Banco MundialBelice).

Thomassiny-Acosta, J. S. (2010). Análisis de los modos de vida de Mahahual y Xcalak y su relación con el estado de conservación del arrecife de coral (Tesis de maestría). El Colegio de la Frontera Sur, Chetumal. 